\title{
SimCompany: An Educational Game Created through a Human-Work Interaction Design Approach
}

\author{
Pedro Campos ${ }^{1}$ and Ana Campos ${ }^{2}$ \\ ${ }^{1}$ University of Madeira, \\ Campus Universitário da Penteada, 9000-390 Funchal, Portugal \\ ISEG School of Economics and Management, Technical University of Lisbon \\ ${ }^{2}$ WowSystems \\ Rua da Alfândega, 78, 3º, 9000-059 Funchal, Portugal \\ pcampos@uma.pt, ana.campos@wowsystems.pt
}

\begin{abstract}
We present a novel children's educational game, which was created through a human-work interaction design - an emerging research field which advocates a better understanding of the relationship between work-domain based empirical studies and iterative design of prototypes and new technologies. We describe how "SimCompany", a game for teaching children about business management concepts, was created. SimCompany proved effective as a teaching tool about business management concepts, and initial evaluation showed a positive increase in students' rate of learning, compared to traditional teaching methods.
\end{abstract}

Keywords: Human work interaction design, sensor-based installations, field observations, educational games, interface design.

\section{Introduction}

In this paper, we describe a novel educational game that uses concepts from HumanWork Interaction Design [1]. This is an emerging research field, which was born under the auspices of IFIP's Working Group 13.6. In a recent workshop [1], new themes and directions of research on human work analysis and design to support it have been outlined. The main target of the work group is the analysis of and the design for the variety of complex work and life contexts found in different business. We argue in this paper that such analysis can be useful when designing childrens' educational games, in particular SimCompany, a game designed and produced with the goal of teaching children management and entrepreneurship concepts.

\section{Related Work}

One good way to motivate children to learn using technology is to apply games, which are well known, exploiting the power of popular TV shows. With the goal of minimizing the amount of effort and requirements to set up a situated learning environment, Lin [2] integrated scenarios of the popular video game Pokemon in classroom education of 2nd grade math concepts. Observations showed that, in such arrangement, they could 
engage some students into the scenarios where math is applied. Since most children inevitably spend much time playing digital games, it is argued that digital game-based learning is one way to involve kids to do the right things with computer [2].

Lee et al. [3] performed a study to investigate whether educational video games could be integrated into a classroom with positive effects for the teacher and students. They conducted the study with 39 2nd grade students using their mathematic drill software "Skills Arena" [3]. Early data from the study suggested that not only do teachers and students enjoy using "Skills Arena", students even exceeded expectations by doing three times more math problems in 19 days than they would have using traditional worksheets. Regardless of the popularity that games exhibit when it comes to teaching children, there is a lack of research towards design approaches that can prove useful when conceiving and designing such games.

\section{3 "SimCompany" and Its Design Process}

"SimCompany" is a fun game designed to instill in children (9-14 years old) the entrepreneurship spirit. As the young player progresses in the game, the basic concepts of consumer behavior, marketing and strategic management are described, illustrated and reinforced in a fun, easy and engaging way.

The goal of the game is to reach the last level and complete it with the greatest amount of points accumulated and before time expires. As an example, we provide a brief description of three levels of this game, taken from the game's script, which was outlined through a human-work design approach.

Level 1: Getting to know the consumer. At the first level, the young player will be faced with a roll of different people (consumers). Based on the description of these characters (age, sex, social class, profession, interests, etc.), the player will have to associate the various products that appear onscreen (e.g. skateboards, neckties, candies, etc.) to the most likely consumer. Figure 1 illustrates this level's user interface.

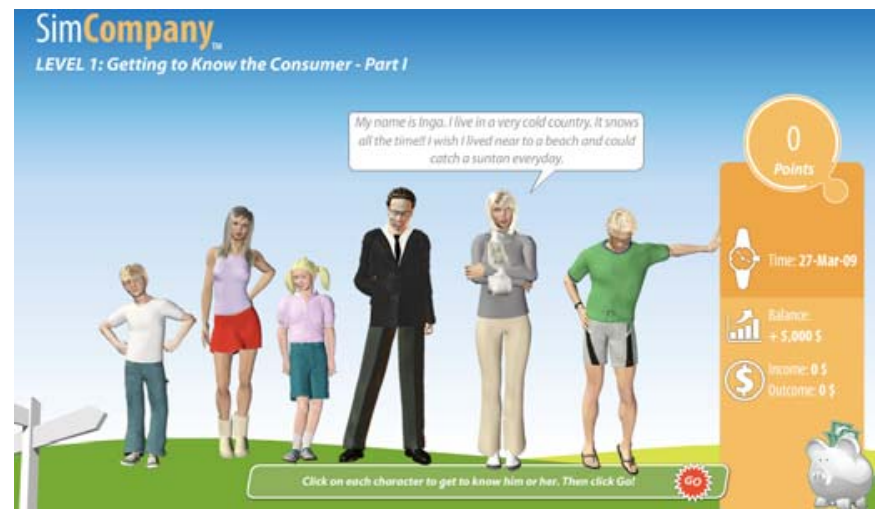

Fig. 1. Overall aspect of the "SimCompany" game, Level 1 
The learning objective is to show the player that there are various factors (demographic, geographic, personal tastes, lifestyle, etc.) that determine the choices of consumption, the ways that each person chooses to spend time and money and that should be taken into account when attempting to open the right business. To move to the next level, the player must accumulate a minimum number of points directly related to the number of correct associations made between consumer-product.

Level 9: Competition. At this level, the competition increases... New organizations start to emerge, which are direct competitors... To worsen the situation there is also an increase on the number of substitute products. The player must be able to identify situations where the threat of substitutes and rivalry in the industry increases and choose the scenario where his/her organization will have better chances to survive. The learning objective is to demonstrate the importance of being aware of the threats of direct competitors and substitute products for the survival of the business.

Level 10: Let's make a trip! The big finale! The business has been a success and now the player has the opportunity to diversify it in geographic terms. But, how? Several scenarios and advice will be given and the player must use his incredible management abilities to internationalize his business in the best possible manner... It will be a difficult task but we know that he will make it...trying is all that matters!

The learning objective of this level is to show children, in very simple and captivating way, the most basic concepts of internationalization of a business.

Human-Work Design Approach. The approach we followed was based on analyzing the work that managers do when conducting their business and from that point we established a creative game script aimed at engaging children but also making them learn the day-to-day real work of a businessman. The approach followed for each of the game's levels was divided into three stages: (i) cognitive analysis, (ii) definition of learning objectives and (iii) translation into a game script.

Stage (i) was directly concerned with outlining the kind of decisions that are made by business managers and entrepreneurs, when leading their operations. Stage (ii) focused on the learning objectives, which were derived after consulting with different business managing experts in the field and also according to some interviews with business leaders and general research literature on the subject. The final stage, translation into a game script, was focused on writing a compelling script that children would enjoy. At the same time, the script would need to meet the learning objectives stated during stage (ii). The final game script document served as a game design document and formed the basis for the user interface design of the game.

\section{Evaluation and Discussion}

The evaluation of this game has been initially conducted with two classes (children aged 9-11) at a local school. Overall there were 28 females and 21 males. In both classes, participants were randomly divided into two groups of equal dimension: a control group, composed of students who took a lesson on managing a business using traditional methods - blackboard, books and the lecture itself - and the experimental group, composed of students who played "SimCompany". Each class was divided into 
three phases: a pretest phase, when students answered a random set of questions about the subject being taught (management) without being taught anything about it. Then came the learning phase itself. It consisted of a traditional lesson in the control group and a "SimCompany" playing session, in the experimental group.

At the end of the class, students performed a similar posttest, so that we could quantify their average degree of learning obtained by each of the groups. One way to measure the degree of learning (how much the students knowledge improved) obtained through a particular mode of education is quantified by the $\langle\mathrm{g}\rangle$ score [4], which is calculated the following way:

$$
<\mathrm{g}>=(\text { posttest } \% \text { - pretest } \%) /(100 \% \text { - pretest } \%)
$$

where posttest $\%$ is the percentage of correct answers in the posttest and pretest $\%$ is the percentage of correct answers in the pretest. The procedure for the control groups was similar, except that the teacher was asked to give the class about the exact same subject but using traditional methods like the whiteboard.

Table 1 shows the evolution from pre-tests to post-tests for each of these groups of students. The results refer to the pre and post-test percentage of correct answers; the right-most column shows the $<\mathrm{g}>$ value.

Table 1. Evolution of the students' degree of learning $<\mathrm{g}>$

\begin{tabular}{llll}
\hline & Pre-Test & Post-Test & $<\mathrm{g}>$ \\
\hline Control Group (without SimCompany) & 59.1 & 70.3 & $27 \%$ \\
Experimental Group (playing SimCompany) & 57.2 & 77.4 & $47 \%$ \\
\hline
\end{tabular}

From this initial evaluation, it became clear that SimCompany is an effective game for teaching children general business management concepts: results showed a higher $<\mathrm{g}>$ value for the experimental group than the control group. This is a step forward in designing technology that really helps children learn. Future work will include evaluating the game in more schools and students, as well as outlining general principles that can prove useful when designing this kind of games. This will also contribute to a growing body of knowledge based on human-work interaction design.

\section{References}

1. Clemmensen, T., Orngreen, R., Pejtersen, A.M.: Describing Users in Contexts: Perspectives on Human-Work Interaction Design. In: Workshop Proceedings of Interact 2005 (2005)

2. Lin, Y.-H.: Integrating Scenarios of Video Games into Classroom Instruction. In: Information Technologies and Applications in Education, ISITAE 2007 (2007)

3. Lee, J., Luchini, K., Michael, B., Norris, C., Soloway, E.: More than just fun and games: assessing the value of educational video games in the classroom. In: CHI 2004 Extended Abstracts on Human Factors in Computing Systems, Vienna, Austria, April 24 - 29 (2004)

4. Mayo, M.J.: Games for science and engineering education. Communications of the ACM 50(7), 30-35 (2007) 